\title{
Effective Diagnosis in Organisation Change Management
}

\author{
Ahmed Hassin \\ Deakin University, Australia
}

\begin{abstract}
Organisations are part of interactive and dynamic environments and modern organisations face considerable pressure to meet or exceed customer/beneficiary's and stakeholders' expectations. The key to establishing effective change and transformation in organisations lies in the early stages of assessment and diagnosis: if diagnosis is wrong, treatment will be ineffective. An organisation development programme should be based on a sound analysis of relevant data about the problem situation, and during the diagnosis process, it is important to look at both the environment and organisation. The OD practitioner's choice of a certain diagnosis method or model or a combination of methods depends on the context, type of problem and organisation.
\end{abstract}

\section{Keywords}

Diagnosis, organisation development, OD practitioner

\section{Introduction}

Organisations do not exist by themselves in a vacuum; they are part of interactive and dynamic environments. In today's highly turbulent, extremely competitive environment, fast communications and technology developments, change of laws, and globalisation, the modern organisation faces considerable pressure to meet or exceed customer/ beneficiary's and stakeholders' expectations by delivering products and services that are of the highest quality.

It is clear that what is needed to survive into this millennium is the ability of organisations (commercial, government or non-government) to respond and adapt. This means competing on several dimensions to meet all customer needs. This will require visionary leadership, and a changeorientation. Organisational results should be the driver to the extent to which the results match customer demands and expectations (Kay and Dayson 1998). The key to establishing an effective change and transformation in organisations lies in the early stages of assessment and diagnosis. It is just like medicine, if diagnosis is wrong, treatment will be ineffective (Bolton and Heap, 2002). "All successful innovation, including effective human factors interventions, needs to address the problems of organizational inertia as well as active opposition and resistance" (Badham, 2006). In any organisation change and development programme, organisation development (OD) practitioners should be aware of where they stand before they plan or conduct any interventions (Wasson, 2004).

Diagnosis provides information that allows a faster-reacting organisation to emerge, one that can deal proactively with changing forces; and, it is the most critical element in the OD process (Brown and Harvey, 2006). Organisations can be conceived of as highly interdependent sub-groups and sub-

Copyright (C) 2010 Victoria University. This document has been published as part of the Journal of Business Systems, Governance and Ethics in both online and print formats. Educational and non-profit institutions are granted a nonexclusive licence to utilise this document in whole or in part for personal or classroom use without fee, provided that correct attribution and citation are made and this copyright statement is reproduced. Any other usage is prohibited without the express permission of the publisher. systems. That is, the impact of any factor in an organisation such as structure, team cohesiveness, leadership, strategy or culture must not be seen in isolation from the factors. The interdependence of these factors and the need for diagnostic tools for their assessment have been extensively discussed in literature. Organisation diagnostic models and surveys have often been demonstrated by OD practitioners to 
be very effective in supporting organisational development programs (Lok and Crawford, 2000). In this review, I have tried to shed the light on the key components of proper and effective diagnosis of problem areas in organisations.

\section{Theoretical Background and Literature Review}

According to Brown and Harvey (2006) organisation diagnosis is a process that helps organisations to improve their capacity to assess and change inefficient patterns of organisational behaviour as a basis for greater effectiveness. An organisation development programme should be based on a sound analysis of relevant data about the problem situation. "Organizational Diagnosis is an effective ways of looking at an organization to determine gaps between current and desired performance and how it can achieve its goals" (RapidBi, 2000-2008). It is very much data-based approach that can set a beginning and the changing objective (Brown and Harvey, 2006). Within the diagnosis and assessment phase, we are trying to uncover essential information about the future in which the organisation must operate and we are beginning to understand the capacity of the organisation to manage its part in the future (Bolton and Heap 2002). Effective diagnosis provides the systematic understanding of the organisation necessary for designing appropriate change interventions intended to resolve problems and improve organisational functioning (Waddell et al, 2004).

Johnston (1979) described a seven-step process for major organisation development efforts. These are:

- Clarification of whole organisation objectives,

- Data gathering and sharing,

- Diagnosis of organisation strengths and weaknesses,

- Joint action prescription of OD interventions to correct weaknesses, -Joint commitment of resources to action,

- Implementation of OD interventions, and

- Periodic progress review of results.

According to Brown and Harvey (2006, p.129), "Diagnosis is a cyclical process that involves data gathering, interpretation and identification of the problem areas and possible action programs".

During the diagnosis process, it is important to look at both the environment and organisation. The environmental factors to be assessed will depend upon the nature of the organisation but will always include cultural factors (Bolton and Heap, 2002). Each organisational culture profile reflects underlying attributes including the management style, strategic plans, climate, reward system, leadership, and basic values of the organisation. So, changing the culture requires that these various elements of culture be identified and altered (Cameron and Quinn, 1999). Organisations try to achieve a sustainable competitive advantage by learning its environment through a scanning process as the environment is a determinant of human resource management (Braton and Gold, 2002). Diagnosing the environment is an assessment process that focuses on determining the readiness of the target group to accept change (Werner and DeSimone, 2006). External environment (economic, social, political, technological, etc) and industry structure are key inputs affecting the strategic planning of an organisation. This Understanding the way how an organisation functions should be done by examining inputs and the alignment of these two components (Waddell et al, 2004). It is important to identify key stakeholders and their views about the organisation (Bolton and Heap, 2002). The first area of diagnosis comprises the interacting sub-elements that compose the organisation, such as: departments, divisions, products and services and the relationship amongst them. The second area is the organisational processes, such as: internal and external communication networks, leadership styles, team conflict resolution, decision-making and planning methods (Brown and Harvey, 2006).

To effectively improve organisational performance, as well as individual and group development, OD practitioners must be knowledgeable of quantitative and qualitative methods, as well as, the different diagnosis models to choose the most appropriate, given the intervention's objectives, resources, and organisational culture and context (Justo, 2009). Mosley and Green (1974) consider the research diagnosis phase (gathering data and studying the organisation) as well as the other OD phases as an important intervention. However, prior to data collection and diagnosis phase of an OD programme, 
there might be some pre-requisites and qualifiers which enable an organisation to succeed in diagnosing problem areas: OD practitioner skills including leadership, project management, communication, problem-solving, interpersonal and personal skills (Brown and Harvey, 2006). Another pre-requisite is a healthy relationship between the organisation and external practitioner. Building a relationship of trust, openness and mutual understanding will make the organisation (or internal practitioners) have real commitment to change. This will, consequently, provide valuable contribution to the diagnosis of the problem areas. The values and ethical beliefs that underline organisation problems suggest that both organisation members and change agents should be jointly involved in discovering the organisational problem areas (Waddell et al, 2004). OD practitioners need maximum involvement and participation of the organisation members in the diagnosis process. An OD practitioner or manager "who spends more time on inspirational or transformational leadership rather than controlling or transactional managerial activities" is a crucial component of effective change management (Badham, 2006). The more objective the data and the more the analysis includes both strengths and weaknesses, the better outcomes the OD programme will have (Brown and Harvey, 2006). Practitioners have to ensure involving people who will be engaged in implementing change initiatives and whose acceptance is necessary for ensuring a successful change effort (Cameron and Quinn, 1999). Lacking the capacity for open discussion, top team cannot arrive at a shared diagnosis (Appelbaum et al, 1998). Diagnosis must be a collaborative venture, so that the process commences with a shared understanding of the basis for subsequent action (Rowley, 2004). Establishing viable system diagnosis requires awareness of reflective thinking and learning as ongoing organisational process (Stephens \& Haslett, 2002).

Organisational diagnostic models are designed to help OD practitioners to categorise data about the organisation, enhance understanding about organisational problems, interpret data systematically and provide appropriate change strategies (Lok and Crawford 2000). Data collection component of the OD process is vital for identification of the problem areas. OD practitioner should acquire relevant, deep and accurate data about the organisation systems (Brown and Harvey, 2006). "To diagnose an organisation, OD practitioners and organisational members need to have some idea as to what information they collect and analyse, which can be based on intuitive hunches right through to scientific explanations of how the organization functions" (Waddell et al, 2004, p.88). Effective information management, including, data quality assurance, training for data collection and input, data warehousing, definition and data analysis, are all essential for effective quality change management (Billing and Temple, 2001).

The failure of organisations to carry out a well planned diagnosis based on accurate data partially explains the high rates of failure of change efforts in organisations (Di Pofi, 2002).

The data collection process has few steps starting with defining the objectives of the change programme. The broad goals and purpose of data collection should be clearly defined to select methods and standards (Brown and Harvey, 2006). It is important to make certain that the target of analysis is the same among all respondents; that is, to be sure that some people are not rating a division while others are looking at the overall organisation (Cameron and Quinn, 1999).

The second step is to identify the key factors involved in the situation such as: culture, isolated management, turnover rates, communication problems, and remuneration schemes. This step should increase the focus on the depth of data to gain insight into other dimensions of the organisation systems (the quality of transactions among individuals and groups) (Brown and Harvey, 2006).

The key to effective diagnosis is to know what to look for at each level (organisation, divisions, departments or teams), as well as how each level affects each other. Understanding organisation level issues is vital in diagnosing any level of analysis as these issues are important inputs to understanding groups or individuals (Waddell et al, 2004).

The third step is selecting a method to collect data depending on the nature of the problem. It should be in a systematic manner to identify certain characteristics that may be measured to help in the achievement of the OD programme (Brown and Harvey, 2006). The first diagnostic tool was presented as the result of a research study conducted in some British companies. The tool is based on interviews, joint working days and some workshops, and it contains nine sub-areas (Moilanen, 2001). Choosing 
an appropriate diagnostic model is very essential; and OD practitioners should be very careful about the model which addresses the organisation's problems as well as ensures comprehensiveness (Waddell et al, 2004). An effective diagnostic model allows identifying reliable data to help organisations better understand their strengths, deficiencies, and opportunities for improvement, to later articulate a targeted intervention and measurement strategy (Justo, 2009). Some major data gathering methods are:

- Secondary Sources of Data, which are generated for other organisational purposes that can be used in identifying problem areas, such as: performance indicators, accounting data, productivity and quality data. Secondary data are recognised of being time and cost-effective as availability of secondary data sources can make the extraction of information rapid and at marginal costs (Sorensen et al, 1996; Steppingstones, 2004). On the other hand, it is also acknowledge that secondary sources have their limitations of availability, or are only available in insufficient quantities (Steppingstones, 2004).

- Direct observation of people behaviours is another important source of data. This can include member actions or reactions to specific situations, and communication patterns. It leads to a greater understanding of the situation and collect more qualitative data (Brown and Harvey, 2006; Stake, 1995). It can be done by site visits to compare the operated behaviour to the observed one. This method provides flexibility and informality of contact (Hill and Stewart, 2000).

- The other method of data collection is Employee Surveys (Brown and Harvey, 2006). The data provide a snapshot of an existing situation, and can be used to compare an organisation's current state with some desired state (Werner and DeSimone, 2006). This method is based on questionnaires used to provide large number of important quantitative information about the values, attitudes and believes of members (Brown and Harvey, 2006). Nowadays, not only traditional paper-based surveys are used; ready-to-use organisational technological solutions are available like web-based surveys (Survey Monkey as an example) (Hartley, 2004). However, if technology-enabled OD solution is selected, the OD practitioner "ensure that you know and define the explicit functional requirements so that your purchase is the best fit possible" (Hartley, 2004).

- Questionnaire-based surveys are one of the most effective tools for OD practitioners to understand and evaluate organisational issues is the (Lok \& Crawford, 2000). As an example, the framework for diagnosis of TQM impact and integration (Kaye and Dyason, 1998) helped companies to identify where they were within the "quality eras" (namely the eras of inspection, quality control, quality assurance or strategic quality management) by comparing their own companies' "characteristics" with those characteristics typically found at each era. While this framework provided an overview and useful starting point, the culture change questionnaire built on the contents of the framework, thereby allowing the issues to be explored in more depth. However, the "heart of quality strategy should be self-evaluation", Billing and Temple (2001) suggest, not the questionnaires.

- Interviews, however, are the most widely used data-gathering technique in OD programmes (Brown\& Harvey, 2006). Interviews can be structured, semi-structured or informal. They are more direct, flexible and not public than surveys (Hill and Stewart, 2000). Interviewing provides data (subjective) that are virtually unobtainable by other methods (Brown\& Harvey, 2006). Interviews or even structured or non-structured one-on-one meetings or with more people can be very effective as they are interesting, less stressful and can reveal information that cannot be obtained by other techniques (Badham, 2006).

- Strengths, Weaknesses, Opportunities and Threats (SWOT) analysis is one of the important tools to help understanding the organisation's internal and external environments helps in diagnosing the gaps and recommendation of possible suitable organisation development programme(s) (NMSU, 2009). SWOT analysis is "a simple framework for generating strategic alternatives from a situation analysis" (NetMBA, 2002-7). Billing and Temple (2001) argue that self-evaluation of the organisation by using SWOT analysis should be the basis of review and further development at all organisational levels. 
- Task analysis is conducted to exactly identify the employee needs to do his/ her job effectively (Blanchard and Thacker, 2007). This technique is a process analysis model used in order to design an effective HRD programmes. It helps in identification of performance discrepancy in the "Process" phase of the model, which will, consequently, lead to identification of the training or non-training needs. There is a need to understand how all employees can deliver constant and consistent high levels of service and how we can design jobs and motivate employees to do this (Johnston, 1999). This method can be more applicable in the manufacturing organisations.

Waclawski and Church (1998: 10) consider all the above methods fall within the framework of research action, which is a data-base systematic quantitative and qualitative data gathering process. This "Data-driven Process Using Action Research" model is attributed to Lewin's view of the organisation change in the 1940's and 1950's (Waclawski and Church, 1998: 10; Herbert, 2009). The strength of Lewin's model, according to Herbert (2009) lies in "its ability to recognize which forces are working within an organization, and developing methods to encourage driving forces while minimizing restraining forces". ( Hartley (2004) has highlighted that as OD practices can be "diverse and entail a variety of underlying practices, multiple systems and tools can support different aspects of OD work". Kimberlin and Winterstein (2008) argue that most data sources involve a greater degree of subjectivity in judgment or other potential sources of error; therefore, it is the researcher's duty to control for known sources of error and to report the reliability and validity. Therefore, Kellehear (1990: 52) argues, that use of watchful methods increase the probability of being careful to protect from having any bias and to have objective data.

Date can be collected using a combination various methods at the same time, such as: interviews, questionnaires, observations, and reading of selected organizational documents (Loftin and Moosbruker, 1982). When the appropriate technique is chosen, data collection must be implemented. Data should be collected from different levels and departments across the organisation (Brown\& Harvey, 2006). Origination, development and promotion of change initiatives from the organisation lower levels can be an effective process of change. In particular, allowing people to participate in the early diagnosis of problems helps to motivate constructive behaviour (O'Brien, 2002).

Analysis of collected data is the other important component of the diagnosis process. The analysis may include comparisons among various organisational divisions and managerial levels. Analysis techniques can vary from simple to highly sophisticate statistical and computerised ones (Brown\& Harvey, 2006).

As the sample has to be large enough to enable generalisation of the results and accuracy of data are important factors in the data collection programmes, there should be criteria to gauge if the data have met the objectives. Brown and Harvey (2006) suggested some criteria for evaluating the effectiveness of data collection: The validity of data, the tome to collect data, the cost of data collection, the organisational culture and norms and the Hawthorne effect.

During the diagnosis process, OD practitioners should be aware of the following warning signals:

- Confidentiality of the information,

- Over-diagnosis (lengthy process impossible to adopt corrective measures),

- Crisis diagnosis (immediate short-term crisis instead of important long-term crisis),

- Threatening and overwhelming diagnosis that might be rejected by the organisation,

- Practitioner's imposed favourite diagnosis regardless of the nature of the problem, and

- Diagnosis of the symptoms rather than underlying problems (Brown and Harvey, 2006).

Therefore, focus should be on the problem causes rather than symptoms.

\section{Conclusion}

The way to solve any organisation problems and change should be through effective diagnosis. Diagnosis of existing organisational problems is the first step to solving them. Diagnosis of problem areas has various models and methods which are used according to the context. It is essential that the 
diagnosis process covers the organisation's internal and external environments. The OD practitioner's choice of a certain diagnosis method or model or a combination of methods depends on the context, type of problem and organisation. Both data collection and analysis can be done using traditional manual or technological methods.

\section{References}

Appelbaum, S.H, St-Pierre, N \& Glavas, W 1998, 'Strategic organizational change: the role of leadership, learning, motivation and productivity', Management Decision, vol.36, no. 5, pp. 289301.

Badham, R. J. 2006, 'Mudanças Not Removalists: Rethinking the Management of Organizational Change', Human Factors and Ergonomics in Manufacturing, vol. 16, no. 3, pp. 229-245.

Billing, D. and Temple P. 2001, Quality Management in central and eastern European universities: a perspective on change management', PERSPECTIVES, vol. 5, no. 4, pp. 111-115.

Bolton, M. and Heap, J. 2002, 'The myth of continuous improvement', Work Study, vol. 51, no. 6, pp. 309-313.

Brown, D. R. and Harvey, D. 2006, An Experiential Approach to Organization Development, 7 e.

Braton and Gold, 2002, Human Resource Management: Theory and Practice, $2^{\text {nd }}$ edition.

Cameron, K. S. and Quinn, R. E. 1999, Diagnosing and Changing Organizational Culture- Based on the Competing Values Framework.

Di Pofi, J. A. 2002, 'Organisational diagnostics: integrating qualitative and quantitative methodology', Journal of Organisational Change Management, vol. 15, no. 2, pp 156-168.

Goulielmos, M. 2005, 'Applying the organizational failure diagnosis model to the study of information systems failure', Disaster Prevention and Management, vol. 14, no. 3, pp. 362-377

Hartley, D. E. 2004, 'OD Wired', Digital Beat, American Society of Training and Development

Herbert, S. 2009, Three Diagnostic Methods, viewed 4/9/2010, < http://www.heberts.net/threediagnostic-models/>

Hill, R. and Stewart, J. 2000, 'Human resource development in small organizations', Journal of European Industrial Training, Vol. 24, No. 2/3/4, pp. 105-117

Johnston, R. W. 1979, 'Starting and Organization Development Effort from Scratch... Seven Steps to Whole Organization Development', Training and Development Journal, pp. 12-22.

Johnston R., 1999, 'Service operations management: return to roots', International Journal of Operations \& Production Management, vol. 19 no. 2, pp. 104-124,

Justo, A. 2009, Organizational Development, viewed 2/9/2010, < http://armandojusto.blogspot.com /2009/04/competency-profiling.html>

Kaye, M. and Dyason, M. 1998,'Training and Development: Harnessing human resources to achieve business excellence', The TQM Magazine, vol. 10, no. 5, pp. 387-396.

Kellehear, A. 1990, Every Student's Guide to Sociology, Nelson, Melbourne, Australia.

Kimberlin C. L, and Winterstein A. G. 2008, 'Validity and reliability of measurement instruments used in research', American Society of Health-System Pharmacists, Dec 1, 2008; vol. 65, pp 22762284 ,

Loftin, R.D. and Moosbruker, J.M. 1982, 'Organization Development Methods in the Management of the Information Systems Function', MIS Quarteriy, September 1982, pp. 15-28.

Lok, P. and Crawford, J. 2000, 'The application of a diagnostic model and surveys in organizational development', Journal of Managerial Psychology, vol. 15, no. 2, pp. 108-125.

Moilanen, R. 2001, 'Diagnostic tools for learning organizations', The Learning Organization, vol. 8, no. 1 , pp. 6-20.

Mosley, D. and Green, T. 1974, 'Nominal grouping as an Organization Development Intervention Technique', Training and Development Journal, pp 30-37

NetMBA.com 2002-2007, SWOT Analysis, viewed 18/9/2009, < $\underline{\text { http://www.quickmba.com }}$ /strategy/swot/ >

New Mexico State University 2009, SWOT, viewed 18/9/2009, $<$ http://cbae.nmsu.edu/ dboje /sbc/pages/page3.html>

O'Brien, J 2002, 'Participation as the key to successful change-a public sector case study', Leadership \& Organization Development Journal, vol. 23, No. 8, pp. 442-455. 
RapidBI 2000-2008, Organizational Diagnosis and Development, viewed 1/9/2010, $<$ http://www.rapidbi.com/created/OrganizationalDiagnosisandDevelopment.html $>$

Rowley, J 2004, 'Researching people and Organizations', Library Management, Vol. 15, No.4/5, pp. 208-214.

Sorensen H T, Sabroe S. and Olsen J. 1996, 'A Framework for Evaluation of Secondary Data Sources for Epidemiological Research', International Journal of Epidemiology, vol. 25, no. 2, pp. 435-442

Stephens, J.R \& Haslett ,T 2002, 'Viable Systems Diagnosis and Organisational Learning', Working paper 8/02, Monash University, Faculty of Business and Economics.

Waclawski, J. and Church A. H. 1998, Designing and Using Organizational Surveys, Gower House, England

Waddell, D M, Cummings, T G \& Worley, C G 2004, Organisation Development \& Change, $2^{\text {nd }}$ ed.

Wasson, R. 2004, 'Five steps to effective change management', IEE Engineering Management, March/ April 2004, pp. 14-15

Werner, J M and DeSimone, R L 2006, Human Resource Development, 4 e. 
